\title{
Fusion through Interpretation
}

\author{
Mark J.L. Orr ${ }^{1,2}$, John Hallam ${ }^{3}$, Robert B. Fisher ${ }^{3}$ \\ 1 Advanced Robotics Research Ltd., University Road, Salford M5 4PP, England \\ 2 SD-Scicon Ltd., Abney Park, Cheadle, Cheshire SK8 2PD, England \\ ${ }^{3}$ Department of Artificial Intelligence, Edinburgh University, Forrest Hill, Edinburgh EH1 2QL, \\ Scotland
}

\begin{abstract}
We discuss two problems in the context of building environment models from multiple range images. The first problem is how to find the correspondences between surfaces viewed in images and surfaces stored in the environment model. The second problem is how to fuse descriptions of different parts of the same surface patch. One conclusion quickly reached is that in order to solve the image-model correspondence problem in a reasonable time the environment model must be divided into parts.
\end{abstract}

\section{Introduction}

In many applications of mobile robots there is a need to construct environment models from data gathered as the environment is explored. Environment models are useful for tasks such as recognising objects, navigating routes and planning the acquisition of new data. It is common to use laser scanning devices which produce range images as a primary source of information. This paper is concerned with some of the problems arising when multiple range images from different viewpoints are fused into a single environment model.

The first stage in processing a range image is to segment it into distinct surface patches. Although real environments possess a proportion of curved surfaces, we assume that the segmented patches are planar with poly-line boundaries. When the environment is really curved, the segmented image will contain a group of smaller planar patches approximating the curved surface. We note that it is difficult to construct fast and reliable segmentation systems for non-planar surfaces. However, while the details are different, the principles of the methods we use also apply to curved surfaces.

The surface descriptions extracted from each image and the descriptions contained in the environment model relate to different coordinate frames. As the robot and the sensor attached to it move about, the relation between the model frame and the image frame changes. Before any data from the image can be added to the model, the image surfaces must be transformed into the model coordinate frame. Surfaces common to both image and model, if there are any, can be used to estimate this transform as long as corresponding pairs can be identified correctly. However, finding these correspondences is complicated by the effects of occlusion which can result in different parts of a surface being visible in different images. Consequently, it is necessary to rely for comparisons on properties, such as relative distance and relative orientation, which are independent of occlusion and frame of reference. The method we use, based on constrained search and hypothesis testing, is discussed in Sect. 3.

Section 4 discusses the problem of updating the description of an environment model 
surface with information from a new description of the same patch. If the existing description is incomplete because of occlusion, the new description may supply information about the 'missing' parts. There is thus a requirement to be able to combine the information from two incomplete descriptions.

Underlying everything is the problem of uncertainty: how to make estimates and take decisions in the presence of sensor noise. Much attention has been given to this subject in recent years and stochastic methods have become the most popular way of handling uncertainty. With these methods, when large numbers of estimates and/or decisions are required, the computational burden can be quite substantial and there may be a need to find ways of improving efficiency. Section 2 below touches on this issue.

A more detailed version of this paper can be found in [8].

\section{Uncertainty}

The type of uncertainty we are talking about is primarily due to noise in the numerical data delivered by sensors. Recently, it has become standard practice in robotics and computer vision $[1,2,9,7]$ to represent uncertainty explicitly by treating parameters as random variables and specifying the first two moments (mean and variance) of their probability distributions (generally assumed to be Gaussian). This permits the use of techniques such as the Extended Kalman Filter for estimation problems, and the Mahalanobis Distance test for making decisions.

The Mahalanobis Test is used to decide whether two estimates are likely to refer to the same underlying quantity. For example, suppose two surface descriptions give area estimates of $(\hat{a}, A)$ and $(\hat{b}, B)$ (the first member of each pair is the mean, the second is the variance). These estimates can be compared by computing the quantity

$$
D_{\mathrm{a}}=\frac{(\hat{a}-\hat{b})^{2}}{A+B}
$$

which has a $\chi^{2}$ distribution. Thus one can choose an appropriate threshold on $D_{\mathrm{a}}$ to test the hypothesis that the surface being described is the same in each case.

The same test is applicable in more complicated situations involving binary relations (between pairs of surfaces) and vector valued parameters. In general, some relation like

$$
g\left(\mathbf{x}_{1}, \mathbf{y}_{1}, \mathbf{x}_{2}, \mathbf{y}_{2}\right)=0
$$

will hold between the true values, $\mathbf{x}_{1}$ and $\mathbf{x}_{2}$, of parameters describing some aspect of two image surfaces and the true values, $\mathbf{y}_{1}$ and $\mathbf{y}_{2}$, of parameters describing the same aspect of two model surfaces - though only if the two pairs correspond. If the parameter estimates are $\left(\hat{\mathbf{x}}_{i}, \mathbf{X}_{i}\right)$ and $\left(\hat{\mathbf{y}}_{i}, \mathbf{Y}_{i}\right), i=1,2$, then, to first order, the mean and variance of $g$ are

$$
\begin{gathered}
\hat{g}=\mathbf{g}\left(\hat{\mathbf{x}}_{1}, \hat{\mathbf{y}}_{1}, \hat{\mathbf{x}}_{2}, \hat{\mathbf{y}}_{2}\right), \\
G=\sum_{i=1}^{2}\left(\frac{\partial g}{\partial \mathbf{x}_{i}} \mathbf{X}_{i}{\frac{\partial g}{\partial \mathbf{x}_{i}}}^{T}+\frac{\partial g}{\partial \mathbf{y}_{i}} \mathbf{Y}_{i} \frac{\partial g}{\partial \mathbf{y}_{i}}\right),
\end{gathered}
$$

(where the Jacobians are evaluated at $\hat{\mathbf{x}}_{i}$ and $\hat{\mathbf{y}}_{i}$ ). To test the hypothesis that the two pairs correspond the Mahalanobis Distance $D=\hat{g}^{2} / G$ is computed and compared with the appropriate $\chi^{2}$ threshold. 
If such measures have to be computed frequently but are usually expected to result in hypothesis rejections (as in interpretation trees - see Sect. 3), there is an efficient method for their calculation. We illustrate this for the case of binary relations for the relative distance of two points $\left(\mathbf{p}_{i}\right.$ and $\left.\mathbf{q}_{i}\right)$ and the relative orientations of two vectors $\left(\mathbf{u}_{i}\right.$ and $\mathbf{v}_{i}$ ). The appropriate functions are, respectively,

$$
\begin{gathered}
g_{\mathrm{d}}=\left(\mathbf{p}_{1}-\mathbf{p}_{2}\right)^{\mathbf{T}}\left(\mathbf{p}_{1}-\mathbf{p}_{2}\right)-\left(\mathbf{q}_{1}-\mathbf{q}_{2}\right)^{T}\left(\mathbf{q}_{1}-\mathbf{q}_{2}\right), \\
g_{\circ}=\mathbf{u}_{1}^{T} \mathbf{u}_{2}-\mathbf{v}_{1}^{T} \mathbf{v}_{2} .
\end{gathered}
$$

Additive terms of the form $\mathbf{x}^{T} \mathbf{A x}$, where $\mathbf{A}$ is a variance matrix and $\mathbf{x}$ is a vector, occur in the expressions for the scalar variances $G_{\mathrm{d}}$ and $G_{0}$. We can use the RayleighRitz Theorem [6] and the fact that variance matrices are positive definite to bound such expressions from above by

$$
\mathbf{x}^{T} \mathbf{A x} \leq \lambda_{\max }(\mathbf{A}) \mathbf{x}^{T} \mathbf{x} \leq \operatorname{trace}(\mathbf{A}) \mathbf{x}^{T} \mathbf{x}
$$

This leads to cheaply calculated upper bounds on $G_{d}$ and $G_{0}$ and corresponding lower bounds on $D_{\mathrm{d}}$ and $D_{\mathrm{o}}$. Since these will usually exceed the thresholds, only in a minority of cases will it be necessary to resort to the full, and more expensive, calculations of the variances.

When the relation holding between the parameters (the function in (1)) is vector valued (as for direct comparisons of infinite plane parameters - Sect. 3) a similar procedure can be used. This avoids the necessity of performing a matrix inverse for every test through the inequality

$$
D=\hat{\mathbf{g}}^{T} \mathbf{G}^{-1} \hat{\mathbf{g}} \geq \frac{\hat{\mathbf{g}}^{T} \hat{\mathbf{g}}}{\operatorname{trace}(\mathbf{G})} .
$$

\section{Finding the Correspondences}

Popular methods for solving correspondence problems include constrained search [5] and generate-and-test [4]. We have adopted a hybrid approach similar to [3] where an interpretation tree searches for consistent correspondences between groups of three surfaces, the correspondences are used to estimate the image-to-model transform, the transform is used to predict the location of all image surfaces in the model, and the prediction is used to test the plausibility of the original three correspondences.

To constrain the search we use a unary relation on surface area, a binary relation on relative orientation of surface normals and a binary relation on relative distance of mid-points (see Sect. 2). For surface patches with occluded boundaries it is necessary to make the variances on area and mid-point position appropriately large. In the case of mid-point position, efficiency is maximised by increasing the uncertainty only in the plane of the surface (so that one of the eigenvalues of the variance matrix, with an eigenvector parallel to the surface normal, is small compared to the other two).

Transforms are estimated from the infinite plane parameters $n$ and $d$ (for any point $\mathbf{x}$ in the plane $\mathbf{n}^{T} \mathbf{x}=d$ where $\mathbf{n}$ is the surface normal). The measurement equation used in the Extended Kalman Filter is

$$
\left[\begin{array}{c}
\mathbf{n}_{\mathrm{m}} \\
d_{\mathrm{m}}
\end{array}\right]-\left[\begin{array}{cc}
\mathbf{R} & 0 \\
\mathbf{t}^{T} \mathbf{R} & 1
\end{array}\right]\left[\begin{array}{l}
\mathbf{n}_{\mathrm{i}} \\
d_{\mathrm{i}}
\end{array}\right]=0
$$


where $\left[\begin{array}{ll}\mathbf{n}_{\mathrm{m}}^{T} & d_{\mathrm{m}}\end{array}\right]^{T}$ and $\left[\begin{array}{ll}\mathbf{n}_{\mathrm{i}}^{T} & d_{\mathrm{i}}\end{array}\right]^{T}$ are the parameter vectors for corresponding model and image planes, $t$ is the translation and $\mathbf{R}$ is the rotation matrix, parameterised by a three component vector equal to the product of the rotation angle and the rotation axis [10].

The transform estimated for each group of three correspondences is used to transform all the image surfaces into model coordinates allowing a direct comparison of positions and orientations. Assuming there is at least one group which leads to a sufficiently large number of further correspondences (hits) to add to the original three, the group with the most is chosen as the correct interpretation. If there is no overlap between image and model, none of the groups will develop more hits than the number expected on the basis of random coincidence (which can be calculated and depends on the noise levels). Moving objects in the scene result in multiple consistent groups with distinct transform estimates.

The time required to find all the consistent triples and calculate the number of hits for each is proportional to a fourth order polynomial in $M$ and $N$ - the number of, respectively, model and image surfaces [8]. The number of consistent triples is proportional to a third order polynomial in $M$ and $N$, all but one of them (in a static scene) coming about by random coincidence. Both also depend on noise levels: as uncertainty increases the search constraints become less efficient, more time is spent searching the interpretation tree and more consistent groups are generated by coincidence. In practice, for noise levels of a few percent and sizes of $M>10^{3}$ and $N>10$, the process is intractable.

A dramatic change can be made by partitioning the environment model into parts and searching for correspondences between the image and each part separately (instead of between the image and the whole model). If there are $P$ parts, the search time is reduced by a factor of $P^{3}$ and the number of spurious solutions by $P^{2}$. Such a partition is sensible because it is unlikely that the robot will be able to simultaneously view surfaces of two different rooms in the same building. The perceptual organization can be carried out as a background recognition process with a generic model of what constitutes a part (e.g. a room model).

\section{Updating the Environment Model}

Updating the infinite plane parameters of a model surface after a correspondence has been found between it and an image surface is relatively straight forward using an Extended Kalman Filter. However, updating the boundary or shape information cannot be achieved in the same manner because it is impossible to describe the boundary with a single random variable. Moreover, because of the possibility of occlusion, the shapes of corresponding surfaces may not be similar at all. The problem has some similarity with the problem of matching strings which have common substrings.

The method we have adopted is again based on finding correspondences but only between small data sets with efficient search constraints, so there is not a combinatorial explosion problem. The features to be matched are the vertices and edges making up the poly-line boundaries of the two surface patches, there being typically about 10 edge features in each. If the two boundary descriptions relate to the same coordinate frame the matching criteria may include position and orientation information as well as vertex angles, edge lengths and edge labels (occluded or unoccluded). In practice, because of the possibility of residual errors in the model-image transform estimate (see Sect. 3), 
we exclude position and orientation information from the matching, calculate a new transform estimate from the matched boundary features and use the old estimate to check for consistency.

The search procedure is seeded by choosing a pair of compatible vertices (similar vertex angles) with unoccluded joining edges, so it relies on there being at least one common visible vertex. A new boundary is then traced out by following both boundaries around. When neither edge is occluded both edges are followed; when one edge is occluded the other is followed; when both edges are occluded the outermost is followed. Uncertainty and over- or under-segmentation of the boundaries may give rise to different possible feature matches (handled by an interpretation tree) but the ordering of features around each boundary greatly constraints the combinatorics. If two unoccluded edges don't overlap, if an occluded edge lies outside an unoccluded one or if the transform estimate is incompatible with the previously estimated image-model transform then the seed vertex match is abandoned and a new one tried. If a vertex match is found which allows the boundary to be followed round right back to the initial vertices, the followed boundary becomes the new boundary of the updated surface. Otherwise, the two boundaries must represent disjoint parts of the same surface and the updated surface acquires both.

\section{Conclusions}

Constrained search (interpretation trees) with stochastic techniques for handling uncertainty can be used to solve both the image-model correspondence problem and the boundary-boundary correspondence problem in order to fuse together multiple range images into a surface-based environment model. The combinatorics of the image-model problem are such that environment models must be divided into small parts if the solution method is to be tractable while the combinatorics of the boundary-boundary problem are inherently well behaved.

\section{References}

1. N. Ayache and O.D. Faugeras. Maintaining representations of the environment of a mobile robot. In Robotics Research 4, pages 337-350. MIT Press, USA, 1988.

2. Y. Bar-Shalom and T.E. Fortmann. Tracking and Data Association. Academic Press, UK, 1988.

3. T.J. Fan, G. Medioni, and R. Nevatia. Recognzing 3-d objects using surface descriptions. IEEE Transactions on Pattern Analysis and Machine Intelligence, 11(11):1140-1157, 1989.

4. O.D. Faugeras and M. Hebert. The representation, recognition, and locating of $3 d$ shapes from range data. International Journal of Robotics Research, 5(3):27-52, 1986.

5. W.E.L. Grimson. Object Recognition by Computer: the Role of Geometric Constraints. MIT Press, USA, 1990.

6. R.A. Horn and C.R. Johnson. Matrix Analysis. Cambridge University Press, USA, 1985.

7. M.J.L. Orr, R.B. Fisher, and J. Hallam. Uncertain reasoning: Intervals versus probabilities. In British Machine Vision Conference, pages 351-354. Springer-Verlag, 1991.

8. M.J.L. Orr, J. Hallam, and R.B. Fisher. Fusion through interpretation. Research Paper 572, Dept. of Artificial Intelligence, Edinburgh University, 1992.

9. J. Porril. Fitting ellipses and predicting confidence using a bias corrected Kalman Filter. Image and Vision Computing, 8(1):37-41, 1990.

10. Z. Zhang and O.D. Faugeras. Determining motion from 3d line segment matches: a comparative study. Image and Vision Computing, 9(1):10-19, 1991. 